International Mathematical Forum, 1, 2006, no. 15, 707-712

\title{
SOME RESULTS \\ ON UNIVERSAL MODULES
}

\author{
Necati Olgun \\ Hacettepe University, Faculty of Science, \\ Department of Mathematics, 06532, Beytepe, Ankara, Turkey \\ nolgun@hacettepe.edu.tr \\ Ali Erdogan \\ Hacettepe University, Faculty of Science, \\ Department of Mathematics, 06532, Beytepe, Ankara, Turkey \\ alier@hacettepe.edu.tr
}

\begin{abstract}
In this work we are basicly interested in some problems related to the universal modules of high order derivations introduced and developed by H. Osborn, R.G. Heyneman, M.E. Sweedler and Y. Nakai.

Firstly we have identify the generators of $\operatorname{Ker} \theta$ where $\theta$ is a map from $\Omega_{n}(R)$ to $\Omega_{1}(R)$ and $R$ is a finitely generated algebra.

Secondy we have characterized the homological dimension of $\Omega_{n}(R)$

for an affine domain presented by $R=\frac{k\left[x_{1}, \ldots, x_{s}\right]}{(f)}$.

Finally we have given some examples.
\end{abstract}

Mathematics Subject Classification: Primary 13N05

Keywords: Universal module, projective module, homological dimension.

\section{Introduction}

Let $R$ be a commutative algebra over an algebraically closed field $k$ with characteristic zero. Let $\Omega_{n}(R)$ and $\delta_{n}: R \rightarrow \Omega_{n}(R)$ denote the universal module of $n$-th order derivations and the canonical $n$-th order $k$-derivation of $R$ respectively.

The pair $\left(\delta_{n}, \Omega_{n}(R)\right)$ has the universal mapping property that for any $R$ module $N$ and any higher derivation $d: R \rightarrow N$ of order $\leq n$ there is a unique $R$-homomorphism $h: \Omega_{n}(R) \rightarrow N$ such that $d=h \delta_{n}$. 
$\Omega_{n}(R)$ is generated by the set $\left\{\delta_{n}(r): \quad r \in R\right\}$. Hence if $R$ is finitely generated $k$-algebra $\Omega_{n}(R)$ will be a finitely generated $R$-module.

Theorem 1.1 Let $R=k\left[x_{1}, \ldots, x_{s}\right]$ be an affine $k$-algebra. Then there exists a short exact sequence of $R$-modules

$$
0 \rightarrow \operatorname{Ker} \theta \rightarrow \Omega_{n}(R) \stackrel{\theta}{\rightarrow} \Omega_{1}(R) \rightarrow 0
$$

such that $\theta\left(\delta_{n}(f)\right)=\delta_{1}(f)$ for all $f \in R$ and $\operatorname{Ker} \theta$ is generated by the set $\left\{\delta_{n}\left(x_{1}^{i_{1}} \ldots . . x_{s}^{i_{s}}\right)-\sum \frac{\partial\left(x_{1}^{i_{1}} \ldots . x_{s}^{i_{s}}\right)}{\partial x_{i}} \delta_{n}\left(x_{i}\right)\right\}$

Proof. By the universal property of $\Omega_{n}(R)$ there exists a unique $R$-module homomorphism $\theta: \Omega_{n}(R) \rightarrow \Omega_{1}(R)$ such that $\theta \delta_{n}=\delta_{1}$ and the following diagram commutes.

$$
\begin{array}{ccc}
R & \stackrel{\delta_{1}}{\longrightarrow} & \Omega_{1}(R) \\
\delta_{n} \downarrow & & \downarrow 1_{\Omega_{1}(R)} \\
\Omega_{n}(R) & \stackrel{\theta}{\longrightarrow} & \Omega_{1}(R)
\end{array}
$$

Since $\theta$ is onto

$$
0 \rightarrow \operatorname{Ker} \theta \rightarrow \Omega_{n}(R) \stackrel{\theta}{\rightarrow} \Omega_{1}(R) \rightarrow 0
$$

is an exact sequence of $R$-modules.

Let $S=\left\{\delta_{n}\left(x_{1}^{i_{1}} \ldots . . x_{s}^{i_{s}}\right)-\sum \frac{\partial\left(x_{1}^{i_{1}} \ldots x_{s}^{i_{s}}\right)}{\partial x_{i}} \delta_{n}\left(x_{i}\right)\right\}$.

Let $N$ be a submodule of $\Omega_{n}(R)$ generated by $S$. Our aim is to show that $\operatorname{Ker} \theta=N$.

Clearly $\operatorname{Ker} \theta \supseteq N$.

Let $p$ be the natural map from $\Omega_{n}(R)$ to $\Omega_{n}(R) / N$. Now $p \delta_{n}$ is a differential operator of order $\leq 1$.

$$
\begin{aligned}
{\left[p \delta_{n}, x_{i}, x_{j}\right](1) } & =\left(\left[p \delta_{n}, x_{i}\right] x_{j}-x_{j}\left[p \delta_{n}, x_{i}\right]\right)(1) \\
& =p \delta_{n}\left(x_{i} x_{j}\right)-x_{i} p \delta_{n}\left(x_{j}\right)-x_{j} p \delta_{n}\left(x_{i}\right)+x_{i} x_{j} p \delta_{n}(1) \\
& =p\left(\delta_{n}\left(x_{i} x_{j}\right)-x_{i} \delta_{n}\left(x_{j}\right)-x_{j} \delta_{n}\left(x_{i}\right)\right) \\
& =0
\end{aligned}
$$

Since $\Omega_{1}(R)$ has the universal property, there exists a unique $\beta: \Omega_{1}(R) \rightarrow$ $\Omega_{n}(R) / N$ such that $p \delta_{n}=\beta \delta_{1}$.

Let $t \in K \operatorname{Ker} \theta$. Then $\theta(t)=0 \Rightarrow \beta \theta(t)=0 \Rightarrow p(t)=0 \Rightarrow$ $t \in N$ and the proof follows. $\diamond$ 
Proposition 1.2 Let $R=k\left[x_{1}, \ldots, x_{s}\right]$ be an affine $k$-algebra. Then there exists a short exact sequence of $R$-modules

$$
0 \longrightarrow \operatorname{Ker} \theta \longrightarrow \Omega_{n+1}(R) \stackrel{\theta}{\longrightarrow} \Omega_{n}(R) \longrightarrow 0
$$

and $\operatorname{Ker} \theta$ is generated by $\left\{\left[\delta_{n+1}, r_{0}, r_{1}, \ldots \ldots, r_{n}\right](1) \mid \quad r_{i} \in R\right\}$.

Proof. By the universal property of $\Omega_{n+1}(R)$ there exists a unique $R$ module homomorphism $\theta: \Omega_{n+1}(R) \longrightarrow \Omega_{n}(R)$ such that $\theta\left(d_{n+1}\right)=d_{n}$ and the following diagram commutes.

$$
\begin{array}{ccc}
R & \stackrel{d_{n}}{\longrightarrow} & \Omega_{n}(R) \\
d_{n+1} \downarrow & & \downarrow 1_{\Omega_{n}(R)} \\
\Omega_{n+1}(R) & \stackrel{\theta}{\longrightarrow} & \Omega_{n}(R)
\end{array}
$$

Since $\theta$ is onto

$$
0 \rightarrow K \operatorname{Ker} \theta \rightarrow \Omega_{n+1}(R) \stackrel{\theta}{\rightarrow} \Omega_{n}(R) \rightarrow 0
$$

is an exact sequence of $R$-modules.

Rest of proof can be seen as in the Theorem 1.1. $\diamond$

\section{Homological Dimension of Universal modules}

Let $I$ be an ideal of a ring $R$. Consider the universal $n$-th order derivative operators $\left(d_{n}, \Omega_{n}(R)\right)$ and $\left(\delta_{n}, \Omega_{n}(R / I)\right)$ for $R$ and $R / I$ respectively. Since the composite of the two maps

$$
R \stackrel{\pi}{\longrightarrow} R / I \stackrel{\delta_{n}}{\longrightarrow} \Omega_{n}(R / I)
$$

is an $n$-th order derivative operator, by universality there exists a unique surjective $\operatorname{map} \beta: \Omega_{n}(R) \longrightarrow \Omega_{n}(R / I)$ such that $\beta d_{n}=\delta_{n} \pi$. Here $\pi$ is the natural map. Now we can define an $R / I$-module homomorphism $\phi: R / I \otimes_{R} \Omega_{n}(R) \longrightarrow$ $\Omega_{n}(R / I)$ by $\phi=1 \otimes \beta$. Hence we have

$$
0 \longrightarrow \frac{\left(N+I \Omega_{n}(R)\right)}{I \Omega_{n}(R)} \longrightarrow \frac{\Omega_{n}(R)}{I \Omega_{n}(R)} \stackrel{\phi}{\longrightarrow} \Omega_{n}(R / I) \longrightarrow 0
$$

an exact sequence of $R / I$-modules. Here $N$ is the submodule of $\Omega_{n}(R)$ generated by the elements of the form $d_{n}(r), r \in I$. We identified $R / I \otimes_{R} \Omega_{n}(R)$ with the quotient $\Omega_{n}(R) /\left(I \Omega_{n}(R)\right)$.

We suppose that $R$ be a polynomial ring $k\left[x_{1}, \ldots ., x_{s}\right]$. Then $\Omega_{n}(R)$ is a free $R$-module of $\operatorname{rank}\left(\begin{array}{c}n+s \\ s\end{array}\right)-1$ with a basis $\left\{d_{n}\left(x^{\alpha}\right)|\quad 0 \leq| \alpha \mid \leq n\right\}$ 
where $x^{\alpha}=x_{1}^{\alpha_{1}}, \ldots . ., x_{s}^{\alpha_{s}}$ and $|\alpha|=\alpha_{1}+\ldots .+\alpha_{s}$.

Let $S$ be a multiplicatively closed subset of $R$. From the fact that $\Omega_{n}(R) \otimes_{R} R_{S} \simeq \Omega_{n}\left(R_{S}\right)$, it follows that $\Omega_{n}\left(R_{S}\right)$ is free of $\operatorname{rank}\left(\begin{array}{c}n+s \\ s\end{array}\right)-1$. Furthermore if $I$ is an ideal of $R$ generated by $f_{1}, \ldots, f_{p}$ then we have the following Lemma.

Lemma 2.1 $\frac{\left(N+I \Omega_{n}(R)\right)}{I \Omega_{n}(R)}$ is generated by the set

$\left\{d_{n}\left(x_{1}^{\alpha_{1}} \ldots x_{t}^{\alpha_{t}} f_{j}\right)+I \Omega_{n}(R) \mid \quad 0 \leq \alpha_{1}+\ldots . .+\alpha_{t} \leq n-1, j=1, . ., p\right\}$

Proof. [1] $\diamond$

Corollary 2.2 $\Omega_{n}(R / I)$ is generated by the set

$$
\left\{d_{n}\left(x^{\alpha}+I\right)|| \alpha \mid \leq n\right\}
$$

where $d_{n}: R / I \longrightarrow \Omega_{n}(R / I)$ is the universal differential operator.

Proof. $[1] \diamond$

Let $R$ be a polynomial ring $k\left[x_{1}, \ldots ., x_{s}\right]$ with an ideal $I$ of $R$ generated by $f \in R$.

Let $S=R / I$. By Corollary 2.2. $\Omega_{n}(S) \simeq F / N$ where $F$ is a free $S$ module on $\left\{d_{n}\left(x^{\alpha}\right)|\quad| \alpha \mid \leq n\right\}$ and $N$ is a submodule of $F$ generated by $\left\{d_{n}\left(x^{\alpha} f\right)|| \alpha \mid<n\right\}$. Therefore we have

$$
0 \longrightarrow N \longrightarrow F \longrightarrow \Omega_{n}(S) \longrightarrow 0
$$

the short exact sequence of S-modules.

Theorem 2.3 Let $S$ be a regular local ring of dimension $s-1$ and $m$ a maximal ideal of $S$. Then $N \nsubseteq m F$.

Proof. Let $S$ be a regular local ring. Then $\Omega_{n}(S)$ is a free $S$-module of $\operatorname{rank}\left(\begin{array}{c}n+s-1 \\ s-1\end{array}\right)-1$. Therefore $\frac{\Omega_{n}(S)}{m \Omega_{n}(S)}$ is a vectorspace of dimension $\left(\begin{array}{c}n+s-1 \\ s-1\end{array}\right)-1$ over $S / m$. If $N \subseteq m F$ then

$$
\frac{\Omega_{n}(S)}{m \Omega_{n}(S)} \simeq \frac{F / N}{m(F / N)} \simeq \frac{F / N}{(m F+N) / N} \simeq \frac{F}{m F}
$$

$\frac{F}{m F}$ which has dimension $\left(\begin{array}{c}n+s \\ s\end{array}\right)-1$ as an $S / m$ vectorspace. This contradiction shows that $\Omega_{n}(S)$ cannot be projective. Hence $N \nsubseteq m F$. 
Corollary 2.4 Let $S$ be a regular ring and $m$ a maximal ideal of $S$. Then $N \nsubseteq m F$.

Theorem 2.5 Let $S=\frac{k\left[x_{1}, \ldots ., x_{s}\right]}{(f)}$ be an affine domain. Then homological dimension of $\Omega_{n}(S)$ is less than or equal to one.

Proof. $[2] \diamond$

Theorem 2.6 Let $S=\frac{k\left[x_{1}, \ldots ., x_{s}\right]}{(f)}$ be an affine domain and $m$ a maximal ideal of $S$. If $N \subseteq m F$ then homological dimension of $\Omega_{n}(S)$ is one.

Proof. From Theorem 2.5. homological dimension of $\Omega_{n}(S)$ is less than or equal to one.

We need to see that $h d\left(\Omega_{n}(S)\right) \geq 1$. Suppose that $\Omega_{n}(S)$ is a projective $S$-module. We can assume that $S$ is a local ring and so $\Omega_{n}(S)$ is free of rank $\left(\begin{array}{c}n+s-1 \\ s-1\end{array}\right)-1$ as the dimension of $S \quad s-1$. If $m$ is the maximal ideal of $S$ then $\frac{\Omega_{n}(S)}{m \Omega_{n}(S)}$ is a vectorspace of dimension $\left(\begin{array}{c}n+s-1 \\ s-1\end{array}\right)-1$ over $S / m$.

If $N \subseteq m F$ then

$$
\frac{\Omega_{n}(S)}{m \Omega_{n}(S)} \simeq \frac{F / N}{m(F / N)} \simeq \frac{F / N}{(m F+N) / N} \simeq \frac{F}{m F}
$$

which has dimension $\left(\begin{array}{c}n+s \\ s\end{array}\right)-1$ as an $S / m$ vectorspace. This contradiction shows that $\Omega_{n}(S)$ cannot be projective. So $h d\left(\Omega_{n}(S)\right)=1 \diamond$

Example 2.7 Let $R=\frac{k[x, y]}{\left(y^{2}-x^{3}\right)}$ be a quotient polynomial ring. For the short exact sequence of $R$-modules

$$
0 \longrightarrow \operatorname{Ker} \theta \longrightarrow \Omega_{3}(R) \stackrel{\theta}{\longrightarrow} \Omega_{1}(R) \longrightarrow 0
$$

$\Omega_{1}(R)$ and $\Omega_{3}(R)$ are generated by $\left\{\delta_{1}(x), \delta_{1}(y)\right\}$ and $\left\{\delta_{3}(x), \delta_{3}(y), \delta_{3}(x y), \delta_{3}\left(x^{2}\right)\right.$, $\left.\delta_{3}\left(x^{2} y\right), \delta_{3}\left(x^{3}\right)\right\}$ respectively.

Hence Ker $\operatorname{K}$ is generated by

$$
\begin{gathered}
\left\{\delta_{3}(x y)-x \delta_{3}(y)-y \delta_{3}(x), \delta_{3}\left(x^{2}\right)-\right. \\
-2 x \delta_{3}(x), \delta_{3}\left(x^{2} y\right)-2 x y \delta_{3}(x)-x^{2} \delta_{3}(y), \delta_{3}\left(x^{3}\right) \\
\left.-3 x^{2} \delta_{3}(y)\right\} .
\end{gathered}
$$


Example 2.8 Let $R=\frac{k[x, y]}{\left(x^{2}+y^{2}-1\right)}$ be a quotient polynomial ring. $R$ is a regular ring. Let $m$ be a maximal ideal in $R$. Let $F$ be a free $R$-module generated by $\left\{\delta_{1}(x), \delta_{1}(y)\right\}$ and $N$ be a submodule of $F$ generated by $\left\{x \delta_{1}(x)+\right.$ $\left.y \delta_{1}(y)\right\}$. We know that $\Omega_{1}(R) \simeq F / N$.

Then $N \nsubseteq m F$.

Example 2.9 $S=\frac{k[x, y]}{\left(y^{2}-x^{3}\right)}$ affine domain with dimension one. One may calculate that projective dimension of $\Omega_{n}(S)$ is one.

Example 2.10 $S=\frac{k[x, y, z]}{\left(z^{2}-x y\right)}$ affine domain with dimension two. One may calculate that projective dimension of $\Omega_{n}(S)$ is one.

\section{References}

[1] Erdogan, A., Differential Operators and Their Universal Modules, Ph.D. Thesis, Universty of Leeds, (1993).

[2] Erdogan, A. and Cimen, N., Projective Dimension of The Universal Modules For The Product of a Hypersurface and Affine T-Space, Comm.Alg. (1999) 27(10), 4737-4741.

[3] Nakai, Y., High Order Derivations, Osaka J. Math. (1970),7,1-27.

[4] Sweedler, M.E. and Heyneman, R.G., Affine Hopf Algebras, J.Algebra (1969), 13, 192-241.

[5] Sweedler, M.E., Groups of Simple Algebras, Publ. Math. (1975) No:44.

[6] Osborn, H., Modules of Differentials I, Math. Ann. (1967),170, 221-244.

Received: April 15, 2005 